\title{
Pregnancy-Related Acute Kidney Injury in the United States: Clinical Outcomes and Health Care Utilization
}

\author{
Silvi Shah ${ }^{\mathrm{a}}$ Karthikeyan Meganathan ${ }^{\mathrm{b}}$ Annette L. Christianson ${ }^{\mathrm{b}}$ \\ Kathleen Harrison ${ }^{a}$ Anthony C. Leonard ${ }^{c}$ Charuhas V. Thakar ${ }^{a, d}$ \\ ${ }^{a}$ Division of Nephrology, Kidney C.A.R.E. (Clinical Advancement, Research and Education) Program, University of \\ Cincinnati, Cincinnati, $\mathrm{OH}, \mathrm{USA}_{;}{ }^{\mathrm{b}}$ Department of Biomedical Informatics, University of Cincinnati, Cincinnati, $\mathrm{OH}$, \\ USA; ' Department of Family and Community Medicine, University of Cincinnati, Cincinnati, OH, USA; ${ }^{\mathrm{d} C i n c i n n a t i ~ V A ~}$ \\ Medical Center, Cincinnati, OH, USA
}

\section{Keywords}

Acute kidney injury · Pregnancy · Race/ethnicity · Mortality

\begin{abstract}
Background: Acute kidney injury (AKI) during pregnancy is a public health problem and is associated with maternal and fetal morbidity and mortality. Clinical outcomes and health care utilization in pregnancy-related AKI, especially in women with diabetes, are not well studied. Methods: Using data from the 2006 to 2015 Nationwide Inpatient Sample, we identified 42,190,790 pregnancy-related hospitalizations in women aged 15-49 years. We determined factors associated with AKI, including race/ethnicity, and associations between AKI and inpatient mortality, and between AKI and cardiovascular (CV) events, during pregnancy-related hospitalizations. We calculated health care expenditures from pregnancy-related AKI hospitalizations. Results: Overall, the rate of AKI during pregnancy-related hospitalizations was $0.08 \%$. In the adjusted regression analysis, a higher likelihood of AKI during pregnancy-related hospitalizations was seen in 2015 (OR 2.20; $95 \% \mathrm{Cl} 1.89-2.55)$ than in 2006; in older women aged $36-40$ years (OR $1.49 ; 95 \% \mathrm{Cl} 1.36-1.64)$ and $41-49$ years (OR $2.12 ; 95 \% \mathrm{Cl} 1.84-2.45)$ than in women aged $20-25$ years; in
\end{abstract}

\begin{tabular}{ll}
\hline KARGER & $\begin{array}{l}\text { ( } 2020 \text { The Author(s) } \\
\text { Published by S. Karger AG, Basel }\end{array}$ \\
karger@karger.com & This article is licensed under the Creative Commons Attribution- \\
www.karger.com/ajn & NonCommercial-NoDerivatives 4.0 International License (CC BY- \\
& NC-ND) (http://www.karger.com/Services/OpenAccessLicense). \\
Usage and distribution for commercial purposes as well as any dis- \\
tribution of modified material requires written permission.
\end{tabular}

blacks (OR 1.52; 95\% Cl 1.40-1.65) and Native Americans (OR $1.45 ; 95 \% \mathrm{Cl} 1.10-1.91)$ than in whites, and in diabetic women (OR 4.43; 95\% Cl 4.04-4.86) than in those without diabetes. Pregnancy-related hospitalizations with AKI were associated with a higher likelihood of inpatient mortality (OR 13.50; 95\% Cl 10.47-17.42) and CV events (OR 9.74; 95\% Cl 9.0810.46) than were hospitalizations with no AKI. The median cost was higher for a delivery hospitalization with AKI than without AKI (USD 18,072 vs. 4,447). Conclusion: The rates of pregnancy-related AKI hospitalizations have increased during the last decade. Factors associated with a higher likelihood of AKI during pregnancy included older age, black and Native American race/ethnicity, and diabetes. Hospitalizations with pregnancy-related AKI have an increased risk of inpatient mortality and CV events, and a higher health care utilization than do those without AKI.

(c) 2020 The Author(s)
Published by S. Karger AG, Basel

\section{Introduction}

Acute kidney injury (AKI) increases the risk of hospital and long-term mortality [1-3]. Moreover, AKI is associated with adverse health outcomes including worsen-

Silvi Shah, MD, MS

Division of Nephrology, University of Cincinnati

231 Albert Sabin Way, MSB 6112

Cincinnati, OH 45267 (USA)

E-Mailshah2sv@ucmail.uc.edu 
ing of kidney function leading to both chronic kidney disease and end-stage kidney disease, and a higher risk of adverse cardiac events $[4,5]$. AKI is not uncommon in children and young adults, but young people remain underrepresented in AKI research [6]. Since pregnancy-related AKI may be especially consequential due to pregnancy's increased demands on kidney function, AKI during pregnancy is an important public health problem.

Despite advances in health care, maternal mortality has increased by $>2$-folds in the United States with the reported pregnancy-related deaths increased to 17.2 deaths per 100,000 live births in 2015 from 7.2 deaths per 100,000 live births in 1987 [7]. Cardiovascular (CV) disease is the leading cause of maternal morbidity and mortality during pregnancy and accounts for one-third of pregnancy-related deaths in the United States [7, 8]. AKI is further associated with higher risks of mortality and of CV events, but there are limited data on the epidemiology of AKI in reproductive-aged women $[4,5]$. Although the incidence of pregnancy-related AKI has decreased in the recent years, the data are mostly from single-center studies, and differences by diabetes status, age, and race/ethnicity are not known. Black, Native American, and Alaska Native women are 2-3 times more likely to die from pregnancy-related causes than are white women, a disparity that increases with women's age, but little is known about racial/ethnic differences in pregnancy-related AKI [9].

Our objective was to assess the trends and factors associated with AKI in hospitalized pregnant women in the United States, to describe their health outcomes of mortality and CV events, and to ascertain the delivery and non-delivery related hospitalization costs. We thus conducted a retrospective cohort study of women with pregnancy-related hospitalizations, using the nationwide inpatient sample from 2006 to 2015, to determine AKI rates and factors associated with AKI, with inpatient mortality, and with CV events. Such factors of interest included age, race/ethnicity, diabetic status, and patterns of health care utilization.

\section{Material and Methods}

\section{Data Sources and Study Population}

We performed a retrospective cohort study to determine rates of AKI during pregnancy in the United States. We used the National Inpatient Sample (NIS), the largest publicly available allpayer inpatient health care database in the United States compiled by the Healthcare Cost and Utilization Project (HCUP). NIS is a nationally representative $20 \%$ stratified sample of US hospital discharges.
We identified 42,190,790 hospitalizations (survey-weighted) from the NIS that occurred in women aged 15-49 years during pregnancy from January 1, 2006 to September 30, 2015, in the United States. Using the International Classification of Diseases, Ninth Revision, Clinical Modification (ICD-9-CM) codes, current procedural terminology, 4 th revision codes, and diagnostic related group codes, we searched for discharge diagnoses and medical procedures indicative of pregnancy, including delivery $[10,11]$. The specificity of this code-based method for establishing the occurrence of pregnancy has been validated in prior studies (online suppl. Table 1; for all online suppl. material, see www.karger.com/ doi/10.1159/000505894). We did not include hospitalizations from October 1, 2015 to December 31, 2019 due to change to ICD-10-CM codes during that period.

All results reported in this study utilized the trend weights provided in the NIS data to obtain weighted national estimates from the unweighted observations. The University of Cincinnati Institutional Review Board committee approved and deemed the study exempt because of the use of de-identified data.

\section{Outcomes and Predictors}

Our clinical outcomes of interest were (1) AKI occurrence during pregnancy-related hospitalization, (2) maternal inpatient mortality during pregnancy-related hospitalizations, and (3) CV events (defined by acute myocardial infarction, congestive heart failure, and/or stroke) during pregnancy-related hospitalizations. AKI and $\mathrm{CV}$ events were identified using ICD-9-CM primary or secondary diagnoses and procedure codes (online suppl. 1). We also calculated delivery and non-delivery associated hospital costs using HCUP's cost-to-charge ratio files. The total cost was inflation-adjusted to year 2015 US dollar equivalent using the annual averages of the Consumer Price Index for All Urban Consumers for inpatient hospital services provided by the US Department of Labor's Bureau of Labor Statistics [12]. Covariates included the following patient demographics and hospital characteristics: age, race/ethnicity, insurance, residential income quartile, hospital location (rural; urban), teaching status (teaching; non-teaching), hospital region (northeast; midwest; south; west), bed size (small; medium; large), admission type (elective; non-elective), delivery, discharge disposition (home; transfer; other), and length of stay. Race/ethnicity was grouped into non-Hispanic white, non-Hispanic black, Hispanic, Asian/Pacific Islander, Native American, and others/unknown. Observations with missing race/ethnicity values $(14.2 \%)$ were grouped in a category with "others" $(4.1 \%)$. Socioeconomic status was determined from the residential income quartile based on the estimated median income associated with patient zip codes. The ICD-9 CM diagnosis codes were used to determine the comorbidity of diabetes mellitus and occurrence of preeclampsia/eclampsia during hospitalizations (online suppl. 1). The Agency for Healthcare Research and Quality Elixhauser comorbidity index for in-hospital mortality was calculated for each hospitalization observation using the indicator variables for 29 comorbidities included in the NIS data and using the weights, methodology and programming code available from HCUP [13]. Observations with missing values for other covariates (Table 1) were excluded from the analyses.

\section{Statistical Analysis}

Descriptive statistics is reported as percentages for categorical variables and as median values and 95\% CIs for continuous variables. Statistical significance was set at a two-tailed $p$ value of 0.05 , 
Table 1. Baseline characteristics of pregnancy-related hospitalizations with and without AKI

\begin{tabular}{|c|c|c|c|}
\hline Characteristic & $\begin{array}{l}\text { Pregnancy-related } \\
\text { non-AKI hospitalizations } \\
(n=42,190,790)\end{array}$ & $\begin{array}{l}\text { Pregnancy-related } \\
\text { AKI hospitalizations } \\
(n=32,385)\end{array}$ & $\begin{array}{l}\text { Rao-Scott modified } \\
\text { chi-square } p \text { value }\end{array}$ \\
\hline \multicolumn{4}{|l|}{ Hospitalization characteristics } \\
\hline Delivery & 90.0 & 42.3 & $<0.001$ \\
\hline \multicolumn{4}{|l|}{ Elective admission (99.6\% } \\
\hline non-missing) & 52.7 & 79.2 & $<0.001$ \\
\hline Year & & & $<0.001$ \\
\hline 2006 & 11.0 & 6.1 & \\
\hline 2007 & 11.6 & 8.5 & \\
\hline 2008 & 10.7 & 8.3 & \\
\hline 2009 & 10.4 & 8.8 & \\
\hline 2010 & 9.8 & 9.9 & \\
\hline 2011 & 9.7 & 9.7 & \\
\hline 2012 & 9.9 & 10.5 & \\
\hline 2013 & 9.7 & 12.2 & \\
\hline 2014 & 9.9 & 14.7 & \\
\hline $2015^{*}$ & 7.4 & 11.4 & \\
\hline \multicolumn{4}{|l|}{ Patient characteristics } \\
\hline Age, years & & & $<0.001$ \\
\hline Median ${ }^{\mathrm{a}}$ & $26.9(26.9-27.0)$ & $28.0(27.7-28.2)$ & \\
\hline $15-19$ & 8.9 & 8.0 & \\
\hline $20-25$ & 28.7 & 25.5 & \\
\hline $26-30$ & 28.4 & 24.8 & \\
\hline $31-35$ & 22.4 & 23.0 & \\
\hline $36-40$ & 9.8 & 13.6 & \\
\hline $41-49$ & 1.9 & 5.1 & \\
\hline Race/ethnicity & & & $<0.001$ \\
\hline White & 44.4 & 33.0 & \\
\hline Black & 12.8 & 29.8 & \\
\hline Hispanic & 19.5 & 15.5 & \\
\hline Asian/Pacific Islander & 4.3 & 3.4 & \\
\hline Native American & 0.7 & 1.0 & \\
\hline Others/unknown & 18.4 & 17.4 & \\
\hline Insurance (99.8\% non-missing) & & & $<0.001$ \\
\hline Medicare/medicaid & 44.4 & 56.0 & \\
\hline Private & 49.2 & 36.7 & \\
\hline Self-pay/no charge/other & 6.4 & 7.4 & \\
\hline \multicolumn{4}{|l|}{ Residential income quartile $(98.1 \%$} \\
\hline non-missing) & & & $<0.001$ \\
\hline First & 28.2 & 38.0 & \\
\hline Second & 25.3 & 24.9 & \\
\hline Third & 24.4 & 21.4 & \\
\hline Fourth & 22.1 & 15.8 & \\
\hline Diabetes mellitus & 1.2 & 12.6 & $<0.001$ \\
\hline Preeclampsia/eclampsia & 4.3 & 29.4 & $<0.001$ \\
\hline Elixhauser comorbidity index & & & $<0.001$ \\
\hline$\leq 0$ & 94.4 & 31.1 & \\
\hline $1-4$ & 2.6 & 7.8 & \\
\hline$\geq 5$ & 3.0 & 61.2 & \\
\hline \multicolumn{4}{|l|}{ Hospital characteristics } \\
\hline Bed size (99.4\% non-missing) & & & $<0.001$ \\
\hline Small & 11.9 & 7.3 & \\
\hline Medium & 27.4 & 22.7 & \\
\hline Large & 60.8 & 70.0 & \\
\hline
\end{tabular}


Table 1. (continued)

\begin{tabular}{|c|c|c|c|}
\hline Characteristic & $\begin{array}{l}\text { Pregnancy-related } \\
\text { non-AKI hospitalizations } \\
(n=42,190,790)\end{array}$ & $\begin{array}{l}\text { Pregnancy-related } \\
\text { AKI hospitalizations } \\
(n=32,385)\end{array}$ & $\begin{array}{l}\text { Rao-Scott modified } \\
\text { chi-square } p \text { value }\end{array}$ \\
\hline \multicolumn{3}{|l|}{ Location (99.4\% non-missing) } & \multirow[t]{4}{*}{$<0.001$} \\
\hline Rural & 10.8 & 3.6 & \\
\hline Urban non-teaching & 38.2 & 24.8 & \\
\hline Urban teaching & 51.1 & 71.6 & \\
\hline \multicolumn{3}{|l|}{ Region } & \multirow[t]{5}{*}{$<0.001$} \\
\hline Northeast & 16.2 & 15.1 & \\
\hline Midwest & 21.2 & 22.8 & \\
\hline South & 38.3 & 42.2 & \\
\hline West & 24.3 & 19.9 & \\
\hline \multicolumn{3}{|l|}{ Discharge (99.98\% non-missing) } & \multirow[t]{7}{*}{$<0.001$} \\
\hline Home (self-care or home health) & 99.19 & 85.58 & \\
\hline \multicolumn{3}{|l|}{ Transfer (short-term hospital or } & \\
\hline other healthcare facility) & 0.52 & 8.48 & \\
\hline Inpatient mortality & 0.01 & 3.98 & \\
\hline Other & 0.28 & 1.96 & \\
\hline Length of stay, days ${ }^{\mathrm{a}, \mathrm{b}}$ & $1.8(1.8-1.8)$ & $5.7(5.5-5.8)$ & \\
\hline \multicolumn{4}{|c|}{$\begin{array}{l}\text { All values represented in percentages except otherwise noted. All results are weighted for the survey design. } \\
\text { * Data for year } 2015 \text { exclude the months October, November, and December. }\end{array}$} \\
\hline
\end{tabular}

unadjusted for multiple tests. We determined the unadjusted rates of AKI overall and by age, race/ethnicity, diabetic status, and socioeconomic status. Rates are expressed as percentages of hospitalizations. The clinical outcomes of AKI, inpatient mortality, and $\mathrm{CV}$ events were analyzed using non-parsimonious multivariable logistic regression models. The regression model predicting AKI was adjusted for covariates of calendar year, age, race/ethnicity, insurance, residential income quartile, delivery, admission type, hospital characteristics (bed size, location, region), Elixhauser mortality score, diabetes, and eclampsia/preeclampsia. An AKI event was the major predictor of interest for the outcomes of inpatient mortality and CV events, and the models were further adjusted non-parsimoniously for AKI. For predicting inpatient mortality, the predictor of CV events was also included. Pregnancyrelated AKI hospitalizations were classified as AKI hospitalizations requiring acute dialysis and AKI hospitalization that did not require acute dialysis, and an additional regression model was performed to study the association of AKI with inpatient mortality by acute dialysis status. The covariates for the logistic regression analyses were chosen based on their known clinical relevance. The risk estimates were expressed as ORs and their 95\% CIs. To account for the survey design involved in the collection of NIS data, surveyspecific procedures (SURVEYFREQ, SURVEYMEANS, SURVEYREG, SURVEYLOGISTIC) in SAS were utilized to obtain estimates of descriptive statistics and of regression parameters. We conducted domain analyses for subpopulations to obtain precise estimates and their variances [14]. All hospitalizations discussed here are hospitalizations of pregnant women. Because we could identify neither women nor individual pregnancies across hospitalizations, all rates are of counts of hospitalizations rather than of women or pregnancies. All analyses were performed using SAS software, version 9.4 (SAS Institute).

\section{Results}

\section{Baseline Demographics and Clinical Characteristics}

A total of 42,190,790 survey-weighted pregnancy-related hospitalizations were identified from 8,730,531 unweighted NIS observations from 2006 to 2015 . The overall rate of AKI in pregnancy-related hospitalizations was $0.08 \%$, and among patients with diabetes it was $0.79 \%$. The median age was 27 years in women without pregnancy-related AKI hospitalization versus 28 years in women with pregnancy-related AKI hospitalizations. Table 1 shows the baseline characteristics in cohorts with and without pregnancy-related AKI hospitalizations. Pregnancy-related hospitalizations with AKI had higher rates of older women, aged $36-40$ years (13.6 vs. 9.8\%) and $41-$ 49 years (5.1 vs. $1.9 \%$ ) compared to non-AKI hospitaliza- 
Fig. 1. Rates of pregnancy-related AKI hospitalizations from 2006 to 2015 by (a) diabetes, (b) age, and (c) race/ethnicity. AKI, acute kidney injury.

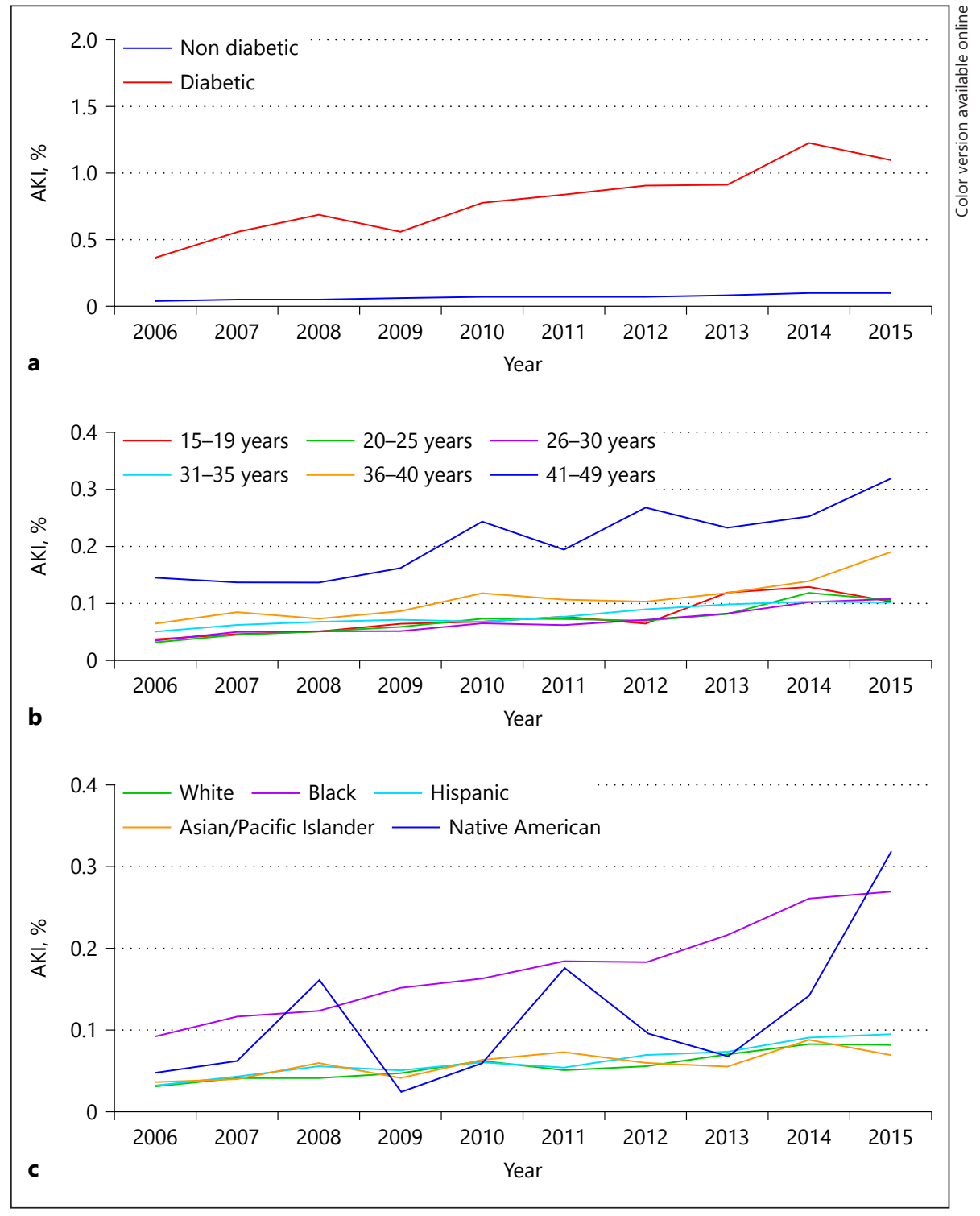

tions. Regarding race/ethnicity, pregnancy-related AKI hospitalizations had a higher proportion of black women than did those without AKI (29.8 vs. $12.8 \%)$. There were a higher proportion of diabetic women among the AKI hospitalizations than in the non-AKI hospitalizations (12.6 vs. 1.2\%). Pregnancy-related AKI hospitalizations had a longer duration of hospital stay than did those without AKI (median 5.7 vs. 1.8 days).

\section{Temporal Trends in Pregnancy-Related AKI and \\ Maternal Mortality}

Overall, the rate of pregnancy-related AKI hospitalizations that included AKI was $0.08 \%$, increasing from $0.04 \%$ in 2006 to $0.12 \%$ in 2015 . Among women with diabetes, the rate of pregnancy-related hospitalizations with AKI increased from $0.36 \%$ in 2006 to $1.10 \%$ in 2015 (Fig. 1a). Regarding age, the rate of AKI in pregnancy-related hospitalizations increased with increasing age (Fig. 1b). Regarding race/ethnicity, the rate of AKI in pregnancy-related hospitalizations was highest in black women $(0.18 \%)$, followed by Native Americans (0.11\%; Fig. 1c). Regarding socioeconomic status, the rate of AKI in pregnancy-related hospitalizations was two-fold higher in patients living in zip codes in the lowest median income quartile than in those living in zip codes in the highest median income quartile ( 0.10 vs. $0.05 \%$; online suppl. 2 ). Urban teaching hospitals had the highest rate of pregnancy-related AKI hospitalizations (0.11\%), followed by ur- 
ban non-teaching hospitals $(0.05 \%)$ and rural hospitals (0.03\%; online suppl. 2).

Inpatient mortality was higher in pregnancy-related AKI hospitalizations (3.98\%) than in pregnancy-related non-AKI hospitalizations $(0.01 \%)$, but did not further differ significantly by diabetic status in pregnancy-related AKI hospitalizations $(3.33 \%$ in diabetic women and $4.08 \%$ in non-diabetic women) and in pregnancy-related non-AKI hospitalizations $(0.02 \%$ in diabetic women vs. $0.01 \%$ in non-diabetic women). The mortality rate in pregnancy-related AKI hospitalizations decreased overall from $4.13 \%$ in 2006 to $2.57 \%$ in 2015 .

\section{Factors Associated with AKI during}

\section{Pregnancy-Related Hospitalizations}

Table 2 shows factors associated with AKI during pregnancy-related hospitalizations. Compared to 2006, the risk of AKI increased consistently through 2015 (OR 2.20; 95\% CI 1.89-2.55). The risk of AKI was increased in older women. Compared to women aged 20-25years, the risk of AKI was higher in women aged 26-30 years (OR 1.09; 95\% CI 1.02-1.18), 31-35 years (OR 1.32; 95\% CI 1.22-1.43), 36-40 years (OR 1.49; 95\% CI 1.36-1.64), and $41-49$ years (OR 2.12; 95\% CI 1.84-2.45). Regarding race/ethnicity, the risk of AKI during pregnancy-related hospitalizations was higher in blacks (OR 1.52; 95\% CI 1.40-1.65) and Native Americans (OR 1.45; 95\% CI $1.10-1.91)$ than in whites. Diabetic women had a 4.4 -fold higher likelihood of developing AKI during pregnancyrelated hospitalization than did women with no history of diabetes (OR 4.43; 95\% CI 4.04-4.86). A history of preeclampsia/eclampsia during a hospitalization was associated with a 6.9-fold higher likelihood of AKI during pregnancy-related hospitalizations (OR 6.90; 95\% CI 6.50-7.33). The risk of AKI during a pregnancy-related hospitalization decreased gradually with improving socioeconomic status in terms of residential income quartile. The Elixhauser comorbidity score was associated with increased odds of pregnancy-related AKI (OR 1.22 per unit increase; 95\% CI 1.22-1.23). Women with private insurance had a lower risk of AKI during pregnancy-related hospitalizations than did women with Medicare/Medicaid (OR 0.74; 95\% CI 0.69-0.79). Hospitalizations involving delivery had a lower risk of AKI than did non-delivery hospitalizations (OR 0.20 ; 95\% CI 0.18 0.21 ).

We also examined the interaction between diabetes and age for its effect on AKI, after accounting for other variables, in a model for the risk of AKI during pregnancy-related hospitalizations. A history of diabetes was also
Table 2. Multivariable logistic regression model showing factors associated with AKI in pregnancy-related hospitalizations

\begin{tabular}{|c|c|c|}
\hline & OR $(95 \% \mathrm{CI})$ & $p$ value \\
\hline Delivery & & $<0.001$ \\
\hline No & Reference & \\
\hline Yes & $0.20(0.18-0.21)$ & \\
\hline Admission type & & $<0.001$ \\
\hline Elective & Reference & \\
\hline Non-elective & $0.56(0.53-0.60)$ & \\
\hline Year & & $<0.001$ \\
\hline 2006 & Reference & \\
\hline 2007 & $1.26(1.07-1.48)$ & \\
\hline 2008 & $1.37(1.16-1.62)$ & \\
\hline 2009 & $1.37(1.18-1.61)$ & \\
\hline 2010 & $1.50(1.28-1.77)$ & \\
\hline 2011 & $1.49(1.28-1.74)$ & \\
\hline 2012 & $1.64(1.42-1.91)$ & \\
\hline 2013 & $1.94(1.68-2.25)$ & \\
\hline 2014 & $2.26(1.96-2.60)$ & \\
\hline 2015 & $2.20(1.89-2.55)$ & \\
\hline Age, years & & $<0.001$ \\
\hline $15-19$ & $0.99(0.89-1.10)$ & \\
\hline $20-25$ & Reference & \\
\hline $26-30$ & $1.09(1.02-1.18)$ & \\
\hline $31-35$ & $1.32(1.22-1.43)$ & \\
\hline $36-40$ & $1.49(1.36-1.64)$ & \\
\hline $41-49$ & $2.12(1.84-2.45)$ & \\
\hline Race/ethnicity & & $<0.001$ \\
\hline White & Reference & \\
\hline Black & $1.52(1.40-1.65)$ & \\
\hline Hispanic & $0.90(0.82-0.99)$ & \\
\hline Asian/Pacific Islander & $1.09(0.93-1.26)$ & \\
\hline Native American & $1.45(1.10-1.91)$ & \\
\hline Others/unknown & $1.22(1.10-1.34)$ & \\
\hline Insurance & & $<0.001$ \\
\hline Medicare/medicaid & Reference & \\
\hline Private & $0.74(0.69-0.79)$ & \\
\hline Self-pay/no charge/other & $0.94(0.84-1.04)$ & \\
\hline Residential income quartile & & $<0.001$ \\
\hline First & Reference & \\
\hline Second & $0.93(0.86-1.00)$ & \\
\hline Third & $0.88(0.81-0.95)$ & \\
\hline Fourth & $0.78(0.71-0.86)$ & \\
\hline Diabetes mellitus & & $<0.001$ \\
\hline No & Reference & \\
\hline Yes & $4.43(4.04-4.86)$ & \\
\hline Preeclampsia/eclampsia & & $<0.001$ \\
\hline No & Reference & \\
\hline Yes & $6.90(6.50-7.33)$ & \\
\hline Elixhauser mortality score & & $<0.001$ \\
\hline 1 Unit & $1.22(1.22-1.23)$ & \\
\hline Hospital bed size & & $<0.001$ \\
\hline Small & Reference & \\
\hline Medium & $1.14(1.01-1.28)$ & \\
\hline Large & $1.46(1.31-1.63)$ & \\
\hline Hospital location & & $<0.001$ \\
\hline Rural & Reference & \\
\hline Urban non-teaching & $2.04(1.74-2.38)$ & \\
\hline Urban teaching & $3.06(2.64-3.56)$ & \\
\hline Hospital region & & 0.020 \\
\hline Northeast & Reference & \\
\hline Midwest & $1.18(1.06-1.31)$ & \\
\hline South & $1.07(0.98-1.18)$ & \\
\hline West & $1.07(0.96-1.19)$ & \\
\hline
\end{tabular}

AKI, acute kidney injury. 
found to be associated with increased risks of AKI during pregnancy-related hospitalizations, varying across different age groups. This additional multivariable logistic model identified a modest interaction between age groups and the co-morbidity of diabetes $(p=0.04)$ such that diabetes mellitus was more strongly associated with AKI in the middle age groups (online suppl. 3).

\section{Factors Associated with Maternal Mortality during}

Pregnancy-Related Hospitalizations

Figure 2 shows factors associated with inpatient mortality during pregnancy-related hospitalizations. Pregnant women who developed AKI during hospitalizations had a significantly higher risk of inpatient mortality than did pregnant women with no AKI (OR 13.50; 95\% CI 10.47-17.42). The likelihood of mortality increased with age in women aged $>30$ years, as compared to women 20-25 years old. Black women (OR 1.55; 95\% CI $1.27-$ 1.89) had a higher risk of maternal mortality than did white women. Women with a history of CV events during hospitalizations had a 2.5-fold higher risk of death (OR 2.57; 95\% CI 2.02-3.26) and women with preeclampsia/ eclampsia had a 1.8-fold higher risk of death (OR 1.88; $95 \%$ CI 1.52-2.32). A history of diabetes was not associated with higher mortality during pregnancy-related hospitalizations. Hospitalizations involving delivery had a lower risk of mortality (OR 0.31 ; 95\% CI $0.26-0.37$ ) than those not involving a delivery.

\section{Pregnancy-Related AKI Hospitalizations by Acute}

Dialysis Status

Of all pregnancy-related AKI hospitalizations, 8.89\% required acute dialysis. The maternal mortality rate was $11.81 \%$ for pregnancy-related AKI requiring acute dialysis and $3.22 \%$ for pregnancy-related AKI hospitalizations that did not require dialysis. Compared to non-AKI pregnancy-related hospitalizations, pregnancy-related AKI requiring acute dialysis had an adjusted odd of 31.94 (95\% CI 20.27-50.31) and pregnancy-related AKI hospitalizations that did not require acute dialysis had an adjusted odd of 11.81 (95\% CI 9.03-15.45).

\section{Associations between Pregnancy-Related AKI and CV Events}

Compared to women without AKI during pregnancyrelated hospitalizations, women with AKI during pregnancy-related hospitalizations had a higher frequency of overall CV events ( 0.85 vs. $20.50 \%$ ), acute myocardial infarction ( 0.01 vs. $1.26 \%)$, congestive heart failure (0.84 vs. $19.46 \%)$, and stroke (0.01 vs. $0.91 \%)$. Figure 3 shows the frequency of CV events among women with and without AKI during pregnancy-related hospitalization. A multivariable logistic model showed that women with AKI during pregnancy-related hospitalizations had a tenfold higher adjusted likelihood of CV events than did women without AKI during pregnancy-related hospitalizations (OR 9.74; 95\% CI 9.08-10.46).

\section{Health Care Utilization}

Table 3 shows the health care utilization and health care expenditures by delivery and occurrence of AKI and $\mathrm{CV}$ event. The median cost and median length of stay was higher for both a delivery hospitalization with AKI (USD 18,072 vs. 4,447 , and 6.2 vs. 1.8 days) and a non-delivery AKI hospitalization (USD 12,241 vs. 3,691) than for those without AKI. A delivery hospitalization with the occurrence of $\mathrm{AKI}$ and a $\mathrm{CV}$ event had a much longer length of stay (8.4 vs. 1.8 days) and a higher cost (USD 26,653 vs. $4,447)$ than those with neither an AKI nor a CV event.

\section{Discussion}

The present study, derived from one of the largest national cohorts in the United States, shows an increase in AKI during pregnancy-related hospitalization across a recent decade from $0.04 \%$ in 2006 to $0.12 \%$ in 2015 , higher health care utilization in pregnancy-related AKI hospitalizations, a 13.5-fold higher risk of mortality with $\mathrm{AKI}$, and a significant association between $\mathrm{AKI}$ and $\mathrm{CV}$ events. The risk of AKI increased with increasing age, and diabetes was associated with a 4.4-fold higher risk of AKI during pregnancy. We found significant racial differences in the incidence of AKI and in inpatient mortality during pregnancy, the risk of AKI being higher in blacks and Native Americans than in white women, and the mortality risk being higher in black women than in white women.

Although the incidence of AKI during pregnancy-related hospitalizations was low, the rates increased almost threefold between 2006 and 2015. This is consistent with an increase in obstetric AKI reported by Mehrabadi et al. [15] that showed an increase in AKI from 2.4 per 10,000 deliveries in 1999-2001 to 6.3 per 10,000 deliveries in 2010-2011. In contrast, reports from developing countries show a decline in the incidence of pregnancy-related AKI in recent years, for example, in India, there was a reduction in pregnancy-related AKI among hospitalized women to $1.5 \%$ in the 2010 s from $15 \%$ in the 1980 s [16]. A similar decreasing trend has been reported from China, 


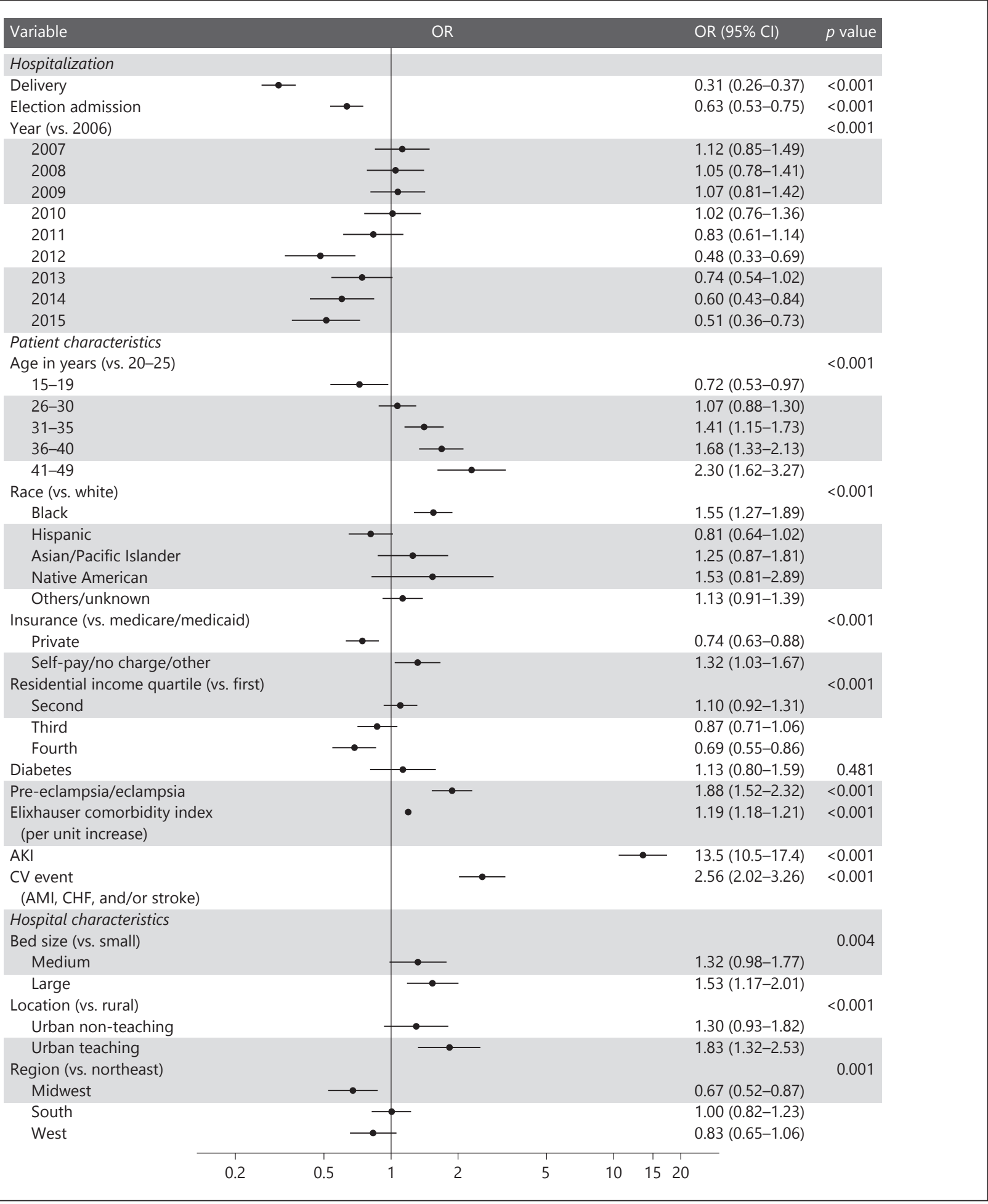

Fig. 2. Multivariable logistic regression model showing factors associated with inpatient mortality in pregnancyrelated hospitalizations. AKI, acute kidney injury; CV, cardiovascular; AMI, acute myocardial infarction; CHF, congestive heart failure. 
Fig. 3. Rates of CV events during pregnancy-related hospitalizations by AKI. CV event defined by occurrence of AMI, CHF and/or stroke. CV, cardiovascular; AKI, acute kidney injury; AMI, acute myocardial infarction; CHF, congestive heart failure.

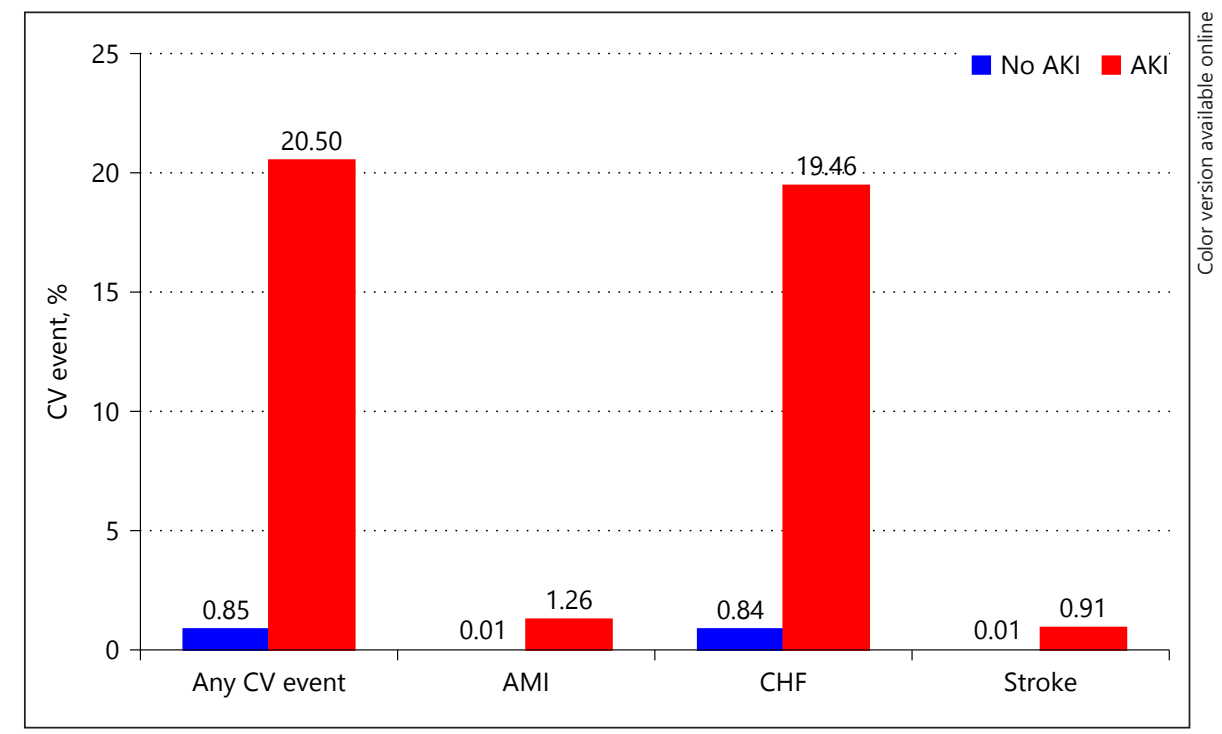

Table 3. Health care utilization and health care expenditures by delivery and occurrence of AKI and CV event

\begin{tabular}{lcc}
\hline & Delivery hospitalization & Non-delivery hospitalization \\
\hline Health care utilization: length of stay ${ }^{\text {, }}$, days & & \\
No AKI, no CV event & $1.8(1.8-1.8)$ & $1.5(1.4-1.5)$ \\
No AKI, yes CV event & $2.6(2.6-2.7)$ & $2.1(2.1-2.2)$ \\
Yes AKI, no CV event & $6.2(6.0-6.4)$ & $4.7(4.5-4.8)$ \\
Yes AKI, yes CV event & $8.4(7.8-9.1)$ & $6.6(6.0-7.2)$ \\
Health care expenditure: cost per hospitalization ${ }^{\text {b }}$, USD & $3,691(3,651-3,730)$ \\
No AKI, no CV event & $4,447(4,403-4,490)$ & $7,713(7,614-7,813)$ \\
No AKI, yes CV event & $6,211(6,112-6,310)$ & $12,241(1,687-12,795)$ \\
Yes AKI, no CV event & $18,072(17,344-18,801)$ & $19,103(17,325-20,881)$ \\
Yes AKI, yes CV event & $26,653(23,996-29,310)$ & \\
\hline & \\
a Median and 95\% CI calculated excluding hospitalizations involving death. & \\
b Median and 95\% CI calculated by converting total charges to costs using the cost to charge ratios provided \\
by HCUP and adjusting for inflation to year 2015 US dollars. \\
AKI, acute kidney injury; CV, cardiovascular; HCUP, Healthcare Cost and Utilization Project. \\
\hline
\end{tabular}

with the incidence of pregnancy-related AKI noted to range from 0.2 to $1.8 \%$ [17]. There are multiple likely explanations for these differences. Of note, the "base rates" of AKI in some of the developing regions have been many times higher than in developed countries, and a reduction in rates may be a true reflection of overall improved medical care. On the other hand, within the United States there may be alternative explanations for the increasing trends. Although antenatal care has improved in the recent years, we speculate that increased awareness and detection of AKI during pregnancy have contributed to the finding of increasing rates of AKI during pregnancy-re- lated hospitalization in the recent years in the United States. Our study showed that both increasing age and the presence of diabetes as a comorbidity was associated with an increased risk of AKI. This was in addition to more traditional or well-known risk factors such as preeclampsia and eclampsia. Given that maternal ages, and associated comorbidities, have increased over time, they may partially explain the increasing frequency of AKI in this patient population. Nevertheless, the present study highlights the need for routine screening of kidney function and the timely detection of AKI in all patients hospitalized during pregnancy. 
Our study finds serious consequences of AKI in this patient population. For instance, AKI was associated with a 13.5-fold risk increase for in-hospital mortality. Although the mortality risk for patients with AKI during pregnancy declined across the years, it was still higher than reported in the prior literature. For example, a meta-analysis concluded that the risk of mortality was 4.5 times higher among 845 pregnancies in 834 women with pregnancy related AKI, compared to 5,387 pregnancies in 5,334 women without AKI [18]. Another important observation from our study was the association between AKI and major CV events during the same hospitalization. Although the study design and availability of data do not allow us to establish a "causeand-effect" relationship between AKI and CV events, it raises the awareness that these 2 events go hand-inhand, and can lead to a significantly higher risk of mortality. Many obstetricians do not routinely check serum creatinine either prior to or early in pregnancy. The present study highlights the need for routine monitoring of kidney function to identify women with AKI, leading to close clinical monitoring and delivery of clinical care aimed at reducing the risk of death and of CV events.

We found significant racial/ethnic differences in the occurrence of AKI and inpatient mortality during pregnancy in the United States. There was a 52\% higher likelihood of AKI during pregnancy among black women and a 45\% higher likelihood among Native American women as compared to white women. While there are no studies examining racial/ethnic differences in AKI for pregnant women, pregnancy rates are highest in black women in the general population, with higher rates for both Native American and black women undergoing dialysis $[19,20]$. Although reasons remain unclear for differences among AKI rates during pregnancy, differential access to obstetric care and level of education could be contributing factors.

Our study has several limitations. First, without clinical information concerning infants, we could not link pregnancy-related AKI to individual pregnancies/women or neonatal outcomes. Second, AKI was ascertained with the use of ICD-9 codes and we were not able to determine stage of AKI, as data on urine output and serum creatinine were not available, probably leading to an underestimation of AKI frequency. Moreover, as comorbidities were inferred from diagnostic codes in the electronic database, prospective cohorts are required to further identify and confirm the risk factors for pregnancy-related AKI. The major strength of our study, however, is its sample, which is large and representative of national pregnancy-related hospitalizations as a whole. The availability of patient-level data permitted a detailed examination of risk factors for AKI in pregnant women and for statistical adjustment for important confounders. The quantification of risk factors' contributions provides stronger tools for preventing AKI at the primary care level.

\section{Conclusion}

The management of AKI during pregnancy is a major clinical challenge because it poses a risk to 2 lives (mother and fetus). Pregnancy-related AKI is associated with an increased risk of mortality and CV events, higher health care utilization, and longer length of hospital stays. The present study increases the awareness of pregnancy-related AKI and suggests improvement to AKI-related care in the United States. The implementation of specific interventions for the prevention, diagnosis and management of AKI in pregnant women may reduce the burden of AKI during hospitalizations in the United States.

\section{Disclosure Statement}

All the authors have no disclosures and competing interests. The results presented in this paper have not been published previously in whole or part, except in abstract format. The Nationwide Inpatient Sample has supplied the data reported here. The interpretation and reporting of these data are the responsibility of the authors and in no way should be seen as official policy or interpretation of the U.S. government.

\section{Funding Sources}

Dr. Silvi Shah is supported by intramural funds from the Division of Nephrology, University of Cincinnati, and the Dialysis Clinic Inc. grant.

\section{Author Contributions}

S.S. initiated the study, designed the study, and wrote the initial manuscript. K.M. contributed to the study design, analyzed and interpreted the data, contributed to the study figures, and reviewed the manuscript. A.L.C., K.H., and A.C.L. contributed to the study design and manuscript review. C.V.T. assisted S.S. with study design and implementation, revision of the manuscript, and did the final approval of the manuscript. All authors reviewed the manuscript. 


\section{References}

1 Heung M, Steffick DE, Zivin K, Gillespie BW, Banerjee T, Hsu CY, et al.; Centers for Disease Control and Prevention CKD Surveillance Team. Acute Kidney Injury Recovery Pattern and Subsequent Risk of CKD: An Analysis of Veterans Health Administration Data. Am J Kidney Dis. 2016 May;67(5):742-52.

2 Pannu N, James M, Hemmelgarn B, Klarenbach S; Alberta Kidney Disease Network. Association between AKI, recovery of renal function, and long-term outcomes after hospital discharge. Clin J Am Soc Nephrol. 2013 Feb;8(2):194-202.

3 Siew ED, Davenport A. The growth of acute kidney injury: a rising tide or just closer attention to detail? Kidney Int. 2015 Jan;87(1):4661.

4 Chawla LS, Amdur RL, Shaw AD, Faselis C, Palant CE, Kimmel PL. Association between $\mathrm{AKI}$ and long-term renal and cardiovascular outcomes in United States veterans. Clin J Am Soc Nephrol. 2014 Mar;9(3):448-56.

5 Thakar CV, Christianson A, Himmelfarb J, Leonard AC. Acute kidney injury episodes and chronic kidney disease risk in diabetes mellitus. Clin J Am Soc Nephrol. 2011 Nov; 6(11):2567-72.

6 McGregor TL, Jones DP, Wang L, Danciu I, Bridges BC, Fleming GM, et al. Acute Kidney Injury Incidence in Noncritically Ill Hospitalized Children, Adolescents, and Young Adults: A Retrospective Observational Study. Am J Kidney Dis. 2016 Mar;67(3):384-90.

7 ACOG Releases Comprehensive Guidance on How to Treat the Leading Cause of U.S. Maternal Deaths: Heart Disease in Pregnancy. 2019. [Accessed October 20, 2019]. Available from: https://http://www.acog.org/AboutACOG/News-Room/News-Releases/2019/ ACOG-Releases-Comprehensive-Guidanceon-How-to-Treat-Heart-Disease-inPregnancy? IsMobileSet=false.

8 Pregnancy Mortality Surveillance System. 2019. [Accessed October 21, 2019]. Available from: https://www.cdc.gov/reproductivehealth/maternalinfanthealth/pregnancy-mortality-surveillance-system.htm?CDC_AA_ refVal=https\%3A\%2F\%2Fwww.cdc.gov\%2Fr eproductivehealth\%2Fmaternalinfanthealth $\% 2$ Fpmss.html

9 Petersen EE, Davis NL, Goodman D, Cox S, Syverson C, Seed K, et al. Racial/Ethnic Disparities in Pregnancy-Related Deaths - United States, 2007-2016. MMWR Morb Mortal Wkly Rep. 2019 Sep 6;68(35):762-5.

10 Wier LM, Witt E, Burgess J, Elixhauser A. Hospitalizations Related to Diabetes in Pregnancy, 2008: Statistical Brief \#102. Healthcare Cost and Utilization Project (HCUP) Statistical Briefs. Rockville (MD): Agency for Healthcare Research and Quality (US); 2006.

11 Kuklina EV, Whiteman MK, Hillis SD, Jamieson DJ, Meikle SF, Posner SF, et al. An enhanced method for identifying obstetric deliveries: implications for estimating maternal morbidity. Matern Child Health J. 2008 Jul; 12(4):469-77.

12 Measuring Price Change in the CPI. Medical care. 2019. [Accessed October 15, 2019]. Available from: https://www.bls.gov/cpi/factsheets/medical-care.htm.

13 Elixhauser Comorbidity Software, Version 3.7. [Accessed July 14, 2019]. Available from: https://www.hcup-us.ahrq.gov/toolssoftware/comorbidity/comorbidity.jsp.

14 Houchens R, Ross D, Elixhauser A. HCUP Methods Series Calculating National Inpatient Sample (NIS) Variances for Data Years 2012 and Later Report. 2015; HCUP Methods Series Report \#2015-09. [Accessed October 15, 2019]. Available from: https://www.hcupus.ahrq.gov/reports/methods/2015_09.jsp.

15 Mehrabadi A, Dahhou M, Joseph KS, Kramer MS. Investigation of a Rise in Obstetric Acute Renal Failure in the United States, 1999-2011. Obstet Gynecol. 2016 May;127(5):899-906.

16 Rao S, Jim B. Acute Kidney Injury in Pregnancy: The Changing Landscape for the 21st Century. Kidney Int Rep. 2018 Feb;3(2):24757.

17 Liu YM, Bao HD, Jiang ZZ, Huang YJ, Wang NS. Pregnancy-related Acute Kidney Injury and a Review of the Literature in China. Intern Med. 2015;54(14):1695-703.

18 Liu Y, Ma X, Zheng J, Liu X, Yan T. Pregnancy outcomes in patients with acute kidney injury during pregnancy: a systematic review and meta-analysis. BMC Pregnancy Childbirth. 2017 Jul;17(1):235

19 Ventura SJ, Curtin SC, Abma JC, Henshaw SK. Estimated pregnancy rates and rates of pregnancy outcomes for the United States, 1990-2008. Natl Vital Stat Rep. 2012 Jun; 60(7):1-21.

20 Shah S, Christianson AL, Meganathan K, Leonard AC, Schauer DP, Thakar CV. Racial Differences and Factors Associated with Pregnancy in ESKD Patients on Dialysis in the United States. J Am Soc Nephrol. 2019 Dec;30(12):2437-48 